\title{
SZEGED INDEX OF A CLASS OF UNICYCLIC GRAPHS
}

\author{
XULI QI \\ Received 13 December, 2018
}

\begin{abstract}
The Szeged index is a modification of the Wiener index to cyclic molecules. The Szeged index of a connected graph $G$ is defined as $S z(G)=\sum_{e \in E(G)} n_{1}(e \mid G) n_{2}(e \mid G)$, where $E(G)$ is the edge set of $G$, and for any $e=u v \in E(G), n_{1}(e \mid G)$ is the number of vertices of $G$ lying closer to vertex $u$ than to vertex $v$, and $n_{2}(e \mid G)$ is the number of vertices of $G$ lying closer to vertex $v$ than to vertex $u$. In this paper, we determine the $n$-vertex unicyclic graphs whose vertices on the unique cycle have degree at least three with the first, the second and the third smallest as well as largest Szeged indices for $n \geq 6, n \geq 7$ and $n \geq 8$, respectively.
\end{abstract}

2010 Mathematics Subject Classification: 05C12; 05C35; 05C90

Keywords: distance, Szeged index, Wiener index, unicyclic graph, fully loaded unicyclic graph

\section{INTRODUCTION}

A topological index is a numerical quantity derived from the hydrogen-depleted graph of a molecule as a tool for compact and effective description of structural formula which allows one to study and predict the structure-property correlations of organic compounds. The Wiener index is one of the oldest topological indices, which was introduced by Wiener [17] in 1947 to study boiling points of paraffins. Since then, it has been used to explain various chemical and physical properties of molecules and to correlate the structure of molecules to their biological activity.

Let $G$ be a simple connected graph with vertex set $V(G)$ and edge set $E(G)$. Let $d_{G}(x, y)$ be the distance between vertices $x$ and $y$ in $G$. Recall that the Wiener index of $G$ is defined as $W(G)=\sum_{\{x, y\} \subseteq V(G)} d_{G}(x, y)[5,8]$.

If $e$ is an edge of $G$ connecting the vertices $u$ and $v$, then we write $e=u v$ or $e=v u$.

Let $e=u v \in E(G)$. Let $n_{1}(e \mid G)$ be the number of vertices of $G$ lying closer to vertex $u$ than to vertex $v$, and $n_{2}(e \mid G)$ be the number of vertices of $G$ lying closer to vertex $v$ than to vertex $u$, i.e.,

$$
n_{1}(e \mid G)=\left|\left\{x \in V(G): d_{G}(x, u)<d_{G}(x, v)\right\}\right|
$$

and

$$
n_{2}(e \mid G)=\left|\left\{x \in V(G): d_{G}(x, v)<d_{G}(x, u)\right\}\right| .
$$

(c) 2019 Miskolc University Press 
If $T$ is a tree, then $W(T)=\sum_{e \in E(T)} n_{1}(e \mid T) n_{2}(e \mid T)$, see [5,8]. Motivated by this equality for Wiener index of trees, Gutman [7] put forward to the Szeged index. For a connected graph $G$, it is defined as [7]

$$
S z(G)=\sum_{e \in E(G)} n_{1}(e \mid G) n_{2}(e \mid G) .
$$

The Szeged index found applications for modeling physicochemical properties as well as physiological activities of organic compounds acting as drugs or possessing pharmacological activity [11], and its mathematical properties may be found, e.g.,[14, 6, 9, 12-16, 19].

A unicyclic graph is a connected graph with a unique cycle. The $n$-vertex unicyclic graphs with the smallest and the largest Szeged indices have been determined [7,16]. Zhou et al. [19] determined the $n$-vertex unicyclic graphs of cycle length $r$ with the smallest and the largest Szeged indices for $3 \leq r \leq n$, the $n$-vertex unicyclic graphs with the second, the third and the fourth smallest Szeged indices, and the $n$-vertex unicyclic graphs with the $k$ th largest Szeged indices for all $k$ up to $\frac{n}{2}+2$ if $n \geq 6$ is even, to four if $n=7$, to five if $n=9$, to $\frac{n+13}{4}$ if $n \equiv 3(\bmod 4)$ with $n \geq 11$, and to $\frac{n+15}{4}$ if $n \equiv 1(\bmod 4)$ with $n \geq 13$.

A unicyclic graph is fully loaded if vertices on its unique cycle all have degree at least three. In the present paper, in continuation of the study on the Szeged index, we determine the $n$-vertex fully loaded unicyclic graphs with the first, the second and the third smallest as well as largest Szeged indices for $n \geq 6, n \geq 7$ and $n \geq 8$, respectively.

\section{PRELIMINARIES}

Let $S_{n}$ and $P_{n}$ be the $n$-vertex star and path, respectively.

Lemma 1 ([5, 8]). Let $T$ be an n-vertex tree different from $S_{n}$ and $P_{n}$. Then

$$
(n-1)^{2}=W\left(S_{n}\right)<W(T)<W\left(P_{n}\right)=\frac{n^{3}-n}{6} .
$$

For a connected graph $G$ with $u \in V(G)$, let $W_{u}(G)=\sum_{v \in V(G)} d_{G}(u, v)$.

Lemma 2 ([18]). Let $T$ be an $n$-vertex tree with $u \in V(T)$, where $u$ is not the center if $T=S_{n}$ and $u$ is not a terminal vertex if $T=P_{n}$. Let $x$ and $y$ be the center of the star $S_{n}$ and a terminal vertex of the path $P_{n}$, respectively. Then

$$
n-1=W_{x}\left(S_{n}\right)<W_{u}(T)<W_{y}\left(P_{n}\right)=\frac{n(n-1)}{2} .
$$

Let $S_{n}^{\prime}$ be the graph formed by attaching a pendent vertex to one pendent vertex of $S_{n-1}$ for $n \geq 4$. Let $P_{n}^{\prime}$ be the graph formed by attaching a pendent vertex to the neighbor of a terminal vertex of $P_{n-1}$ for $n \geq 4$. 
Lemma 3 ([10,18]). Among the $n$-vertex trees with $n \geq 4, S_{n}^{\prime}$ is the unique graph with the second smallest Wiener index, which is equal to $n^{2}-n-2$, and $P_{n}^{\prime}$ is the unique graph with the second largest Wiener index, which is equal to $\frac{1}{6}\left(n^{3}-7 n+18\right)$.

The following lemma may be easily checked.

Lemma 4. Let $T$ be an $n$-vertex tree with $n \geq 5, u \in V(T), T \neq S_{n}, P_{n}$, where $u$ is not a vertex of maximal degree if $T=S_{n}^{\prime}$, and not the terminal vertex whose neighbor has degree two if $T=P_{n}^{\prime}$. Let $x$ be the vertex of maximal degree of $S_{n}^{\prime}$, and $y$ be the terminal vertex whose neighbor has degree two in $P_{n}^{\prime}$. Then $n=W_{x}\left(S_{n}^{\prime}\right)<$ $W_{u}(T)<W_{y}\left(P_{n}^{\prime}\right)=\frac{(n+1)(n-2)}{2}$.

Let $C_{r}\left(T_{1}, T_{2}, \ldots, T_{r}\right)$ be the graph constructed as follows. Let the vertices of the cycle $C_{r}$ be labeled consecutively by $v_{1}, v_{2}, \ldots, v_{r}$. Let $T_{1}, T_{2}, \ldots, T_{r}$ be vertexdisjoint trees such that $T_{i}$ and the cycle $C_{r}$ share exactly one common vertex $v_{i}$ for $i=1,2, \ldots, r$. Denote $|G|=|V(G)|$ for a graph $G$. Then any $n$-vertex unicyclic graph $G$ with a cycle on $r$ vertices is of the form $C_{r}\left(T_{1}, T_{2}, \ldots, T_{r}\right)$, where $\sum_{i=1}^{r}\left|T_{i}\right|=$ $n$. For the graph $G=C_{r}\left(T_{1}, T_{2}, \ldots, T_{r}\right)$, let $d_{i j}=d_{C_{r}}\left(v_{i}, v_{j}\right)$ and $t_{i}=\left|T_{i}\right|$ for $i=1,2, \ldots, r$. Then let $N_{s}=\sum_{i \neq s} t_{i} d_{i s}$.

For a subset $M$ of $E(G), G-M$ denotes the graph obtained from $G$ by deleting the edges in $M$. For a subset $M^{*}$ of the edge set of the complement of $G, G+M^{*}$ denotes the graph obtained from $G$ by adding the edges in $M^{*}$.

Lemma 5 ([19]). Let $G=C_{r}\left(T_{1}, T_{2}, \ldots, T_{r}\right)$ and $T_{i}$ be a star with center $v_{i}$ for $i=1,2, \ldots, r$. Suppose that trees $T_{k}$ and $T_{l}$ are nontrivial. Let $w \in V\left(T_{l}\right)$ with $w \neq v_{l}$. If $r$ is odd and $N_{k}+\frac{1}{2} t_{k} \leq N_{l}+\frac{1}{2} t_{l}$, or $r$ is even and $N_{k} \leq N_{l}$, then $S z\left(G-\left\{v_{l} w\right\}+\left\{v_{k} w\right\}\right)<S z(G)$.

Let $\varepsilon_{n}=1$ if $n$ is odd and $\varepsilon_{n}=0$ if $n$ is even.

Lemma 6 ([9]). Let $G=C_{r}\left(T_{1}, T_{2}, \ldots, T_{r}\right)$. Then

$$
S z(G)=\sum_{i=1}^{r} W\left(T_{i}\right)+\sum_{i=1}^{r}\left(|G|-t_{i}\right) W_{v_{i}}\left(T_{i}\right)+\sum_{i=1}^{r} \sum_{j=1}^{r} t_{i} t_{j} d_{i j}-\varepsilon_{r} \sum_{i<j} t_{i} t_{j} .
$$

For $3 \leq r \leq\left\lfloor\frac{n}{2}\right\rfloor$, let $\mathbb{U}_{n, r}$ be the set of all fully loaded unicyclic graphs with $n$ vertices and unique cycle $C_{r}$. For $n \geq 6$, let $\mathbb{U}_{n}$ be the set of $n$-vertex fully loaded unicyclic graphs.

\section{Fully LOAded UNiCyClic GRAPHS With SMALl SZEGEd INDiCES}

For $3 \leq r \leq\left\lfloor\frac{n}{2}\right\rfloor$, let $S_{n, r}=C_{r}\left(T_{1}, T_{2}, \ldots, T_{r}\right)$ with $T_{1}=\cdots=T_{r-1}=P_{2}$ and $T_{r}=S_{n-2(r-1)}$ with center $v_{r}$. 
Proposition 1. Let $G \in \mathbb{U}_{n, r}$ with $3 \leq r \leq\left\lfloor\frac{n}{2}\right\rfloor$. Then

$$
S z(G) \geq \begin{cases}n^{2}+\left(r^{2}-3 r\right) n-r^{3}+2 r^{2} & \text { if } r \text { is odd } \\ n^{2}+\left(r^{2}-r-1\right) n-r^{3}+r & \text { if } r \text { is even }\end{cases}
$$

with equality if and only if $G=S_{n, r}$.

Proof. By the definition of the Szeged index, if $r$ is odd, then

$$
\begin{aligned}
S z\left(S_{n, r}\right) & =1 \cdot(n-1) \cdot(n-r)+(r-1) \cdot(r-1) \\
& +(r-1) \cdot(n-r+1-2) \cdot(r-1) \\
& =n^{2}+\left(r^{2}-3 r\right) n-r^{3}+2 r^{2},
\end{aligned}
$$

and if $r$ is even, then

$$
\begin{aligned}
S z\left(S_{n, r}\right) & =1 \cdot(n-1) \cdot(n-r)+r \cdot(n-r) \cdot r \\
& =n^{2}+\left(r^{2}-r-1\right) n-r^{3}+r .
\end{aligned}
$$

Let $G=C_{r}\left(T_{1}, T_{2}, \ldots, T_{r}\right)$ be a graph with the smallest Szeged index among graphs in $\mathbb{U}_{n, r}$. We need only to show that $G=S_{n, r}$.

By Lemmas 1, 2 and 6, $T_{i}$ is a star with center $v_{i}$ for $i=1,2, \ldots, r$. Suppose that $t_{k}, t_{l} \geq 3$ with $k \neq l$. Let $w \in V\left(T_{l}\right)$ with $w \neq v_{l}$. Suppose without loss of generality that $N_{k}+\frac{1}{2} t_{k} \leq N_{l}+\frac{1}{2} t_{l}$ if $r$ is odd and that $N_{k} \leq N_{l}$ if $r$ is even. By Lemma 5, $S z\left(G-\left\{v_{l} w\right\}+\left\{v_{k} w\right\}\right)<S z(G)$, a contradiction. Thus there can not be two trees of $T_{1}, T_{2}, \ldots, T_{r}$ with at least three vertices in $G$, i.e., $G=S_{n, r}$.

Let $\Gamma_{n}$ be the set of graphs $C_{3}\left(T_{1}, T_{2}, T_{3}\right)$ in $\mathbb{U}_{n}$ with $\left|T_{1}\right|=\left|T_{2}\right|=2$. Let $\Psi_{n}$ be the set of graphs $C_{3}\left(T_{1}, T_{2}, T_{3}\right)$ in $\mathbb{U}_{n}$ with $\left|T_{3}\right| \geq\left|T_{2}\right| \geq \max \left\{\left|T_{1}\right|, 3\right\}$. Let $\Phi_{n}$ be the set of graphs in $\mathbb{U}_{n}$ with cycle length at least four. Then $\mathbb{U}_{n}=\Gamma_{n} \cup \Psi_{n} \cup \Phi_{n}$.

For $n \geq 7$, let $B_{n}^{\prime}$ be the graph in $\Gamma_{n}$ formed by attaching a path $P_{2}$ and $n-7$ pendent vertices to the vertex of degree two in $C_{3}\left(P_{2}, P_{2}, P_{1}\right)$.

Lemma 7. Among the graphs in $\Gamma_{n}$ with $n \geq 7, B_{n}^{\prime}$ is the unique graph with the second smallest Szeged index, which is equal to $n^{2}+n-12$.

Proof. The case $n=7$ is trivial. Let $G=C_{3}\left(T_{1}, T_{2}, T_{3}\right) \in \Gamma_{n}$ with $n \geq 8$. Note that $\left|T_{1}\right|=\left|T_{2}\right|=2$. By Lemma 6, we have

$$
\begin{aligned}
S z(G) & =1+1+W\left(T_{3}\right)+n-2+n-2+4 W_{v_{3}}\left(T_{3}\right) \\
& +2[2 \cdot 2+2 \cdot(n-4)+2 \cdot(n-4)] \\
& -[2 \cdot 2+2 \cdot(n-4)+2 \cdot(n-4)] \\
& =6 n-14+W\left(T_{3}\right)+4 W_{v_{3}}\left(T_{3}\right),
\end{aligned}
$$

which, together with Lemmas 3 and 4, implies that $B_{n}^{\prime}$ is the unique graph in $\Gamma_{n}$ with the second smallest Szeged index, which is equal to $n^{2}+n-12$. 
Let $S_{n}(a, b, c)=C_{3}\left(T_{1}, T_{2}, T_{3}\right)$ with $\left|T_{1}\right|=a,\left|T_{2}\right|=b,\left|T_{3}\right|=c, a+b+c=n$, and $a, b, c \geq 2$, where $T_{1}\left(T_{2}, T_{3}\right.$, respectively) is a star with center $v_{1}\left(v_{2}, v_{3}\right.$, respectively). Let $G_{n}^{\prime}$ for $n \geq 8$ be the graph obtained from $S_{n-1}(2,3, n-6)$ by attaching a pendent vertex to a pendent vertex at $v_{3}$, and $G_{n}^{\prime \prime}$ for $n \geq 9$ the graph obtained from $S_{n-1}(2,2, n-5)$ by attaching a pendent vertex to the pendent vertex at $v_{2}$.

Lemma 8. Among the graphs in $\Psi_{n}, S_{n}(2,3, n-5)$ for $n \geq 8$ is the unique graph with the smallest Szeged index, which is equal to $n^{2}+n-16 ; G_{8}^{\prime}$ for $n=8, S_{9}(3,3,3)$ for $n=9$, and $S_{n}(2,4, n-6)$ for $n \geq 10$ are the unique graphs with the second smallest Szeged index, which is equal to 61 for $n=8,75$ for $n=9$, and $n^{2}+2 n-25$ for $n \geq 10$.

Proof. For $n \geq 8$, let $G=C_{3}\left(T_{1}, T_{2}, T_{3}\right) \in \Psi_{n}$ with $c \geq b \geq \max \{a, 3\}$, where $\left|T_{1}\right|=a,\left|T_{2}\right|=b,\left|T_{3}\right|=c$ and $a+b+c=n$.

If in $G, T_{1}=P_{2}$ and $T_{2}=S_{3}$ with $v_{2}$ its center, then by Lemma $6, S z(G)=8 n-$ $22+W\left(T_{3}\right)+5 W_{v_{3}}\left(T_{3}\right)$, which, together with Lemmas 1-4, implies that, among the graphs $C_{3}\left(T_{1}, T_{2}, T_{3}\right) \in \Psi_{n}$ with $T_{1}=P_{2}$ and $T_{2}=S_{3}$ with $v_{2}$ its center, $S_{n}(2,3, n-$ $5)$ and $G_{n}^{\prime}$ are the unique graphs with the smallest Szeged index $n^{2}+n-16$ and the second smallest Szeged index $n^{2}+2 n-19$, respectively.

For $n=8$, the graphs in $\Psi_{8}$ are $S_{8}(2,3,3), G_{8}^{\prime}$ and $C_{3}\left(P_{2}, P_{3}, P_{3}\right)$ with $v_{2}$ being a terminal vertex of one $P_{3}$ and $v_{3}$ a terminal vertex of the other $P_{3}$, where $S z\left(S_{8}(2,3,3)\right)=56<S z\left(G_{8}^{\prime}\right)=61<S z\left(C_{3}\left(P_{2}, P_{3}, P_{3}\right)\right)=66$. Thus $S_{8}(2,3,3)$ and $G_{8}^{\prime}$ are the unique graphs in $\Psi_{8}$ respectively with the smallest and the second smallest Szeged indices, which are equal to 56 and 61, respectively.

For $n=9$, by Lemmas 1, 2 and 6, the graphs in $\Psi_{9}$ with the smallest Szeged index are just the graphs $S_{9}(2,3,4)$ and $S_{9}(3,3,3)$ with smaller Szeged index, which is equal to $\min \{74,75\}=74$. Thus $S_{9}(2,3,4)$ is the unique graph in $\Psi_{9}$ with the smallest Szeged index, which is equal to 74 . By the proof at the beginning, the graphs in $\Psi_{9}$ with the second smallest Szeged index are just the graphs in $G_{9}^{\prime}, G_{9}^{\prime \prime}$ and $S_{9}(3,3,3)$ with the smallest Szeged index, which is equal to $\min \{80,80,75\}=75$. Thus $S_{9}(3,3,3)$ is the unique graph in $\Psi_{9}$ with the second smallest Szeged index, which is equal to 75 .

For $n \geq 10$, suppose first that $G=S_{n}(a, b, c)$ and $G \neq S_{n}(2,3, n-5), S_{n}(2,4, n-$ 6). Since $N_{1}=\sum_{i \neq 1} t_{i} d_{i 1}=b+c, N_{2}=\sum_{i \neq 2} t_{i} d_{i 2}=a+c$ and $N_{3}=\sum_{i \neq 3} t_{i} d_{i 3}=$ $a+b$, it is easily seen that $N_{3}+\frac{1}{2} c \leq N_{2}+\frac{1}{2} b \leq N_{1}+\frac{1}{2} a$. By Lemmas 5 and 6 , if $a=2$, then

$$
\begin{aligned}
S z\left(S_{n}(2, b, c)\right) & >S z\left(S_{n}(2,4, n-6)\right)=n^{2}+2 n-25 \\
& >S z\left(S_{n}(2,3, n-5)\right)=n^{2}+n-16,
\end{aligned}
$$


and if $a \geq 3$, then

$$
S z\left(S_{n}(a, b, c)\right) \geq S z\left(S_{n}(3,3, n-6)\right)=n^{2}+2 n-24>n^{2}+2 n-25 .
$$

If $G \neq S_{n}(a, b, c)$, then by the proof at the beginning and applying Lemmas 1,2 and 6, we have either $S z(G) \geq S z\left(G_{n}^{\prime}\right)=S z\left(G_{n}^{\prime \prime}\right)=n^{2}+2 n-19>n^{2}-2 n-25$ for $a=2$, or $S z(G)>S z\left(S_{n}(a, b, c)\right) \geq S z\left(S_{n}(3,3, n-6)\right)>n^{2}+2 n-25$ for $a \geq 3$. Thus, for $n \geq 10, S_{n}(2,3, n-5)$ and $S_{n}(2,4, n-6)$ are the unique graphs with the smallest and the second smallest Szeged indices, which are equal to $n^{2}+n-16$ and $n^{2}+2 n-25$, respectively. The result follows.

Theorem 1. Among the graphs in $\mathbb{U}_{n}$,

(i) $S_{n, 3}$ for $n \geq 6$ is the unique graph with the smallest Szeged index, which is equal to $n^{2}-9$;

(ii) $B_{7}^{\prime}$ for $n=7$, and $S_{n}(2,3, n-5)$ for $n \geq 8$ are the unique graphs with the second smallest Szeged index, which is equal to 44 for $n=7$, and $n^{2}+n-16$ for $n \geq 8$;

(iii) $B_{8}^{\prime}$ for $n=8, S_{9}(3,3,3)$ for $n=9, S_{n}(2,4, n-6)$ for $10 \leq n \leq 12, S_{13}(2,4,7)$ and $B_{13}^{\prime}$ for $n=13$, and $B_{n}^{\prime}$ for $n \geq 14$ are the unique graphs with the third smallest Szeged index, which is equal to 60 for $n=8,75$ for $n=9, n^{2}+2 n-25$ for $10 \leq n \leq$ 12,170 for $n=13$, and $n^{2}+n-12$ for $n \geq 14$.

Proof. By Proposition 1, if $r$ is odd, then

$$
\begin{aligned}
S z\left(S_{n, r+1}\right)-S z\left(S_{n, r}\right) & =(4 r-1) n-5 r^{2}-2 r \\
& \geq(4 r-1) \cdot 2(r+1)-5 r^{2}-2 r \\
& =3 r^{2}+4 r-2>0
\end{aligned}
$$

for $3 \leq r \leq\left\lfloor\frac{n}{2}\right\rfloor-1$, implying that $S z\left(S_{n, r+1}\right)>S z\left(S_{n, r}\right)$, and

$$
\begin{aligned}
S z\left(S_{n, r+2}\right)-S z\left(S_{n, r}\right) & =(4 r-2) n-6 r^{2}-4 r \\
& \geq(4 r-2) \cdot 2(r+2)-6 r^{2}-4 r \\
& =2 r^{2}+8 r-8>0
\end{aligned}
$$

for $3 \leq r \leq\left\lfloor\frac{n}{2}\right\rfloor-2$, implying that $S z\left(S_{n, r+2}\right)>S z\left(S_{n, r}\right)$. If $r$ is even, then

$$
S z\left(S_{n, r+1}\right)-S z\left(S_{n, r}\right)=-n-r^{2}+1<0
$$

for $4 \leq r \leq\left\lfloor\frac{n}{2}\right\rfloor-1$, implying that $S z\left(S_{n, r+1}\right)<S z\left(S_{n, r}\right)$, and

$$
\begin{aligned}
S z\left(S_{n, r+2}\right)-S z\left(S_{n, r}\right) & =(4 r+2) n-6 r^{2}-12 r-6 \\
& \geq(4 r+2) \cdot 2(r+2)-6 r^{2}-12 r-6 \\
& =2 r^{2}+8 r+2>0
\end{aligned}
$$

for $4 \leq r \leq\left\lfloor\frac{n}{2}\right\rfloor-2$, implying that $S z\left(S_{n, r+2}\right)>S z\left(S_{n, r}\right)$. It follows that $S z\left(S_{n, r+1}\right)>S z\left(S_{n, r}\right)$ for odd $r$ with $3 \leq r \leq\left\lfloor\frac{n}{2}\right\rfloor-1, S z\left(S_{n, r+1}\right)<S z\left(S_{n, r}\right)$ for even $r$ with $4 \leq r \leq\left\lfloor\frac{n}{2}\right\rfloor-1$, and $S z\left(S_{n, r}\right)$ is increasing with respect to odd 
$r \in\left\{3,5, \ldots,\left\lfloor\frac{n}{2}\right\rfloor\right\}$ and even $r \in\left\{4,6, \ldots,\left\lfloor\frac{n}{2}\right\rfloor\right\}$. Then by Proposition $1, S_{n, 3}$ for $n \geq 6$ is the unique graph with the smallest Szeged index, which is equal to $n^{2}-9$, proving (i). Moreover, $S_{n, 4}$ for $n=8,9$ and $S_{n, 5}$ for $n \geq 10$ are the unique graphs in $\Phi_{n}$ with the smallest Szeged index, which are equal to $n^{2}+11 n-60$ and $n^{2}+10 n-75$, respectively.

Now we prove $(i i)$. The case $n=7$ is trivial. For $n \geq 8$, the graphs in $\mathbb{U}_{n}$ with the second smallest Szeged index are just the graphs in $\mathbb{U}_{n} \backslash\left\{S_{n, 3}\right\}=\left(\Gamma_{n} \backslash\left\{S_{n, 3}\right\}\right) \cup$ $\Psi_{n} \cup \Phi_{n}$ with the smallest Szeged index, which, by Lemmas 7 and 8, is equal to

$$
\begin{gathered}
\min \left\{S z\left(B_{n}^{\prime}\right), S z\left(S_{n}(2,3, n-5)\right), S z\left(S_{n, 4}\right)\right\} \\
=\min \left\{n^{2}+n-12, n^{2}+n-16, n^{2}+11 n-60\right\}=n^{2}+n-16
\end{gathered}
$$

for $n=8,9$, and

$$
\begin{gathered}
\min \left\{S z\left(B_{n}^{\prime}\right), S z\left(S_{n}(2,3, n-5)\right), S z\left(S_{n, 5}\right)\right\} \\
=\min \left\{n^{2}+n-12, n^{2}+n-16, n^{2}+10 n-75\right\}=n^{2}+n-16
\end{gathered}
$$

for $n \geq 10$. Then (ii) follows.

From $(i)$ and $(i i)$ and by Lemmas 7 and 8 , we find that the graphs in $\mathbb{U}_{n}$ for $n \geq 8$ with the third smallest Szeged index are just the graphs in

$$
\mathbb{U}_{n} \backslash\left\{S_{n, 3}, S_{n}(2,3, n-5)\right\}=\left(\Gamma_{n} \backslash\left\{S_{n, 3}\right\}\right) \cup\left(\Psi_{n} \backslash\left\{S_{n}(2,3, n-5)\right\} \cup \Phi_{n}\right.
$$

for $n \geq 8$, which is equal to

$$
\min \left\{S z\left(B_{8}^{\prime}\right), S z\left(G_{8}^{\prime}\right), S z\left(S_{8,4}\right)\right\}=\min \{60,61,92\}=60
$$

for $n=8$,

$$
\min \left\{\operatorname{Se}\left(B_{9}^{\prime}\right), S z\left(S_{9}(3,3,3)\right), S z\left(S_{9,4}\right)\right\}=\min \{78,75,120\}=75
$$

for $n=9$, and

$$
\begin{aligned}
& \min \left\{S z\left(B_{n}^{\prime}\right), S z\left(S_{n}(2,4, n-6)\right), S z\left(S_{n, 5}\right)\right\} \\
= & \min \left\{n^{2}+n-12, n^{2}+2 n-25, n^{2}+10 n-75\right\}
\end{aligned}
$$

i.e., $n^{2}+2 n-25$ for $10 \leq n \leq 12, n^{2}+2 n-25=n^{2}+n-12=170$ for $n=13$, and $n^{2}+n-12$ for $n \geq 14$. Now (i i i ) follows easily.

\section{FUlly LOADED UNiCyCliC GRAPHS With LARGE SZEGED INDiCES}

For $3 \leq r \leq\left\lfloor\frac{n}{2}\right\rfloor$, let $P_{n, r}=C_{r}\left(T_{1}, T_{2}, \ldots, T_{r}\right)$ with $T_{1}=\cdots=T_{r-1}=P_{2}$ and $T_{r}=P_{n-2(r-1)}$ with a terminal vertex $v_{r}$.

Proposition 2. Let $G \in \mathbb{U}_{n, r}$ with $3 \leq r \leq\left\lfloor\frac{n}{2}\right\rfloor$. Then

$$
S z(G) \leq \begin{cases}\frac{1}{6}\left[n^{3}+\left(-6 r^{2}+12 r-7\right) n+10 r^{3}-24 r^{2}+14 r\right] & \text { if } r \text { is odd } \\ \frac{1}{6}\left[n^{3}+\left(-6 r^{2}+24 r-13\right) n+10 r^{3}-36 r^{2}+20 r\right] & \text { if } r \text { is even }\end{cases}
$$

with equality if and only if $G=P_{n, r}$. 
Proof. By the definition of the Szeged index, if $r$ is odd, then

$$
\begin{aligned}
S z\left(P_{n, r}\right) & =1 \cdot(n-1) \cdot r+2 \cdot(n-2)+\cdots+(n-2 r+1) \cdot(2 r-1) \\
& +(r-1) \cdot(r-1)+(r-1) \cdot(n-r+1-2) \cdot(r-1) \\
& =\frac{1}{6}\left[n^{3}+\left(-6 r^{2}+12 r-7\right) n+10 r^{3}-24 r^{2}+14 r\right],
\end{aligned}
$$

and if $r$ is even, then

$$
\begin{aligned}
S z\left(P_{n, r}\right) & =1 \cdot(n-1) \cdot r+2 \cdot(n-2)+\cdots+(n-2 r+1) \cdot(2 r-1)+r \cdot(n-r) \cdot r \\
& =\frac{1}{6}\left[n^{3}+\left(-6 r^{2}+24 r-13\right) n+10 r^{3}-36 r^{2}+20 r\right] .
\end{aligned}
$$

Let $G=C_{r}\left(T_{1}, T_{2}, \ldots, T_{r}\right)$ be a graph with the largest Szeged index among graphs in $\mathbb{U}_{n, r}$. We need only to show that $G=P_{n, r}$.

By Lemmas 1, 2 and 6, $T_{i}$ is a path with a terminal vertex $v_{i}$ for each $i=1,2, \ldots, r$. Then by Lemma 6, we have

$$
\begin{aligned}
S z(G) & =\sum_{i=1}^{r} \frac{1}{6}\left(t_{i}^{3}-t_{i}\right)+\sum_{i=1}^{r} \frac{1}{2}\left(n-t_{i}\right) t_{i}\left(t_{i}-1\right)+\sum_{i=1}^{r} \sum_{j=1}^{r} t_{i} t_{j} d_{i j}-\varepsilon_{r} \sum_{i<j} t_{i} t_{j} \\
& =-\frac{1}{3} \sum_{i=1}^{r} t_{i}^{3}+\frac{1}{2}(n+1) \sum_{i=1}^{r} t_{i}^{2}-\frac{1}{2} n^{2}-\frac{1}{6} n+\sum_{i=1}^{r} \sum_{j=1}^{r} t_{i} t_{j} d_{i j}-\varepsilon_{r} \sum_{i<j} t_{i} t_{j} .
\end{aligned}
$$

Suppose that there exist distinct $k, l, m$ with $1 \leq k, l, m \leq r$, such that $t_{k}, t_{l}, t_{m} \geq 3$ and $t_{m} \geq \max \left\{t_{k}, t_{l}\right\}$.

Case 1. $r$ is odd. Assume that $t_{k}^{2}+(n+1) t_{l}+2 N_{l} \leq t_{l}^{2}+(n+1) t_{k}+2 N_{k}$. Let $G^{\prime}$ be the graph formed from $G$ by deleting the pendent vertex in $T_{l}$ and attaching it to the pendent vertex in $T_{k}$. Obviously, $G^{\prime} \in \mathbb{U}_{n, r}$. Note that $2 t_{l}+2 d_{k l}<t_{l}+t_{m}+r \leq$ $n<n+2$. Then

$$
\begin{aligned}
& S z(G)-S z\left(G^{\prime}\right) \\
& =\left(t_{k}^{2}+n t_{l}+2 N_{l}\right)-\left(t_{l}^{2}+n t_{k}+2 N_{k}\right)+2 t_{l}+2 d_{k l}-n-1+\left(-t_{k}+t_{l}-1\right) \\
& =\left[t_{k}^{2}+(n+1) t_{l}+2 N_{l}\right]-\left[t_{l}^{2}+(n+1) t_{k}+2 N_{k}\right]+2 t_{l}+2 d_{k l}-(n+2)<0,
\end{aligned}
$$

and thus $S z(G)<S z\left(G^{\prime}\right)$, which is a contradiction. Thus $r-2$ of $t_{1}, t_{2}, \ldots, t_{r}$ are equal to 2 , say $t_{i}=2$ for $i \neq k, l$. Let $t_{k}=a$ and $t_{l}=b$. We write $G=G_{a, b}$, where $a, b \geq 2$ and $a+b=n-2(r-2)$. Note that $N_{k}=2 W_{v_{k}}\left(C_{r}\right)+(b-2) d_{k l}$ and $N_{l}=$ $2 W_{v_{l}}\left(C_{r}\right)+(a-2) d_{k l}$. If $a \geq b \geq 3$, then since that $a+b+2 d_{k l}<a+b+r \leq n+1$ and $2 b+2 d_{k l}<a+b+r<n+2$, we have

$$
\begin{aligned}
& S z\left(G_{a, b}\right)-S z\left(G_{a+1, b-1}\right) \\
& =\left[a^{2}+(n+1) b+4 W_{v_{l}}\left(C_{r}\right)+2(a-2) d_{k l}\right] \\
& -\left[b^{2}+(n+1) a+4 W_{v_{k}}\left(C_{r}\right)+2(b-2) d_{k l}\right]+2 b+2 d_{k l}-(n+2)
\end{aligned}
$$




$$
=(a-b)\left[a+b+2 d_{k l}-(n+1)\right]+2 b+2 d_{k l}-(n+2)<0,
$$

implying that $S z\left(G_{a, b}\right)$ is maximum for $a \geq b$ and $a+b=n-2(r-2)$ if and only if $a=n-2(r-1)$ and $b=2$. It follows that $G=P_{n, r}$.

Case 2. $r$ is even. Assume that $t_{k}^{2}+n t_{l}+2 N_{l} \leq t_{l}^{2}+n t_{k}+2 N_{k}$. Let $G^{\prime}$ be the graph formed from $G$ by deleting the pendent vertex in $T_{l}$ and attaching it to the pendent vertex in $T_{k}$. Obviously, $G^{\prime} \in \mathbb{U}_{n, r}$. Note that $2 t_{l}+2 d_{k l} \leq t_{l}+t_{m}+r \leq n<n+1$. Then

$$
S z(G)-S z\left(G^{\prime}\right)=\left(t_{k}^{2}+n t_{l}+2 N_{l}\right)-\left(t_{l}^{2}+n t_{k}+2 N_{k}\right)+2 t_{l}+2 d_{k l}-(n+1)<0,
$$

and thus $S z(G)<S z\left(G^{\prime}\right)$, which is a contradiction. Thus $r-2$ of $t_{1}, t_{2}, \ldots, t_{r}$ are equal to 2 , say $t_{i}=2$ for $i \neq k, l$. Let $t_{k}=a$ and $t_{l}=b$. We write $G=G_{a, b}$, where $a, b \geq 2$ and $a+b=n-2(r-2)$. Note that $N_{k}=2 W_{v_{k}}\left(C_{r}\right)+(b-2) d_{k l}$ and $N_{l}=$ $2 W_{v_{l}}\left(C_{r}\right)+(a-2) d_{k l}$. If $a \geq b \geq 3$, then since that $a+b+2 d_{k l} \leq a+b+r \leq n$ and $2 b+2 d_{k l} \leq a+b+r<n+1$, we have

$$
\begin{aligned}
& S z\left(G_{a, b}\right)-S z\left(G_{a+1, b-1}\right)=\left[a^{2}+n b+4 W_{v_{l}}\left(C_{r}\right)+2(a-2) d_{k l}\right] \\
& -\left[b^{2}+n a+4 W_{v_{k}}\left(C_{r}\right)+2(b-2) d_{k l}\right]+2 b+2 d_{k l}-(n+1) \\
& =(a-b)\left(a+b+2 d_{k l}-n\right)+2 b+2 d_{k l}-(n+1)<0,
\end{aligned}
$$

implying that $S z\left(G_{a, b}\right)$ is maximum for $a \geq b$ and $a+b=n-2(r-2)$ if and only if $a=n-2(r-1)$ and $b=2$. It follows that $G=P_{n, r}$.

By combining Cases 1 and 2, the result follows.

Let $P_{n}(a, b, c, d)=C_{4}\left(T_{1}, T_{2}, T_{3}, T_{4}\right)$ with $\left|T_{1}\right|=a,\left|T_{2}\right|=b,\left|T_{3}\right|=c,\left|T_{4}\right|=d$, $a+b+c+d=n$, and $a, b, c, d \geq 2$, where $T_{1}\left(T_{2}, T_{3}, T_{4}\right.$, respectively) is a path with a terminal vertex $v_{1}\left(v_{2}, v_{3}, v_{4}\right.$, respectively). Let $P_{n, 4}^{\prime}$ be the graph formed by attaching a pendent vertex to the neighbor of the pendent vertex of $T_{4}$ in $P_{n-1,4}$.

Lemma 9. Among the graphs in $\mathbb{U}_{n, 4}$ with $n \geq 9, S_{9,4}$ for $n=9$ and $P_{n}(2,3,2, n-$ 7) for $n \geq 10$ are the unique graphs with the second largest Szeged index, which is equal to 120 for $n=9$, and $\frac{1}{6}\left(n^{3}-19 n+198\right)$ for $n \geq 10 ; P_{n}(2,2,3, n-7)$ for $n=10,11, P_{n}(2,4,2, n-8)$ for $12 \leq n \leq 16, P_{17}(2,4,2,9)=P_{17,4}^{\prime}$ for $n=17$, and $P_{n, 4}^{\prime}$ for $n \geq 18$ are the unique graphs with the third largest Szeged index, which is equal to $\frac{1}{6}\left(n^{3}-31 n+306\right)$ for $n=10,11, \frac{1}{6}\left(n^{3}-25 n+264\right)$ for $12 \leq n \leq 16,792$ for $n=17$ and $\frac{1}{6}\left(n^{3}-19 n+162\right)$ for $n \geq 18$.

Proof. The case $n=9$ is trivial. Let $G=C_{4}\left(T_{1}, T_{2}, T_{3}, T_{4}\right) \in \mathbb{U}_{n, 4}$ with $n \geq 10$.

Suppose first that there is exactly one tree, say $T_{4}$ with more than two vertices. Note that $t_{1}=t_{2}=t_{3}=2$. By Lemma 6 , we have $S z(G)=19 n-67+W\left(T_{4}\right)+$ $6 W_{v_{4}}\left(T_{4}\right)$. In this case, by Lemmas 3 and 4 , we know that $P_{n, 4}^{\prime}$ is the unique graph with the second largest Szeged index, which is equal to $\frac{1}{6}\left(n^{3}-19 n+162\right)$. 
Suppose that there are two trees $\left(T_{3}\right.$ and $T_{4}$, or $T_{2}$ and $\left.T_{4}\right)$ with more than two vertices. By the proof of Proposition 2, we have

$$
S z(G) \leq S z\left(P_{n}\left(2,2, t_{3}, t_{4}\right)\right) \leq S z\left(P_{n}(2,2,3, n-7)\right)=\frac{1}{6}\left(n^{3}-31 n+306\right)
$$

or

$$
S z(G) \leq S z\left(P_{n}\left(2, t_{2}, 2, t_{4}\right)\right) \leq S z\left(P_{n}(2,3,2, n-7)\right)=\frac{1}{6}\left(n^{3}-19 n+198\right) .
$$

If there are three or four trees with more than two vertices, then by the proof of Proposition 2, we have

$S z(G) \leq S z\left(P_{n}\left(t_{1}, t_{2}, t_{3}, t_{4}\right)\right) \leq \max \left\{S z\left(P_{n}(2,2,3, n-7)\right), S z\left(P_{n}(2,3,2, n-7)\right)\right\}$.

It follows that the graphs in $\mathbb{U}_{n, 4}$ with the second largest Szeged index are just the graphs in $\mathbb{U}_{n, 4} \backslash\left\{P_{n, 4}\right\}$ with the largest Szeged index, which is equal to

$$
\begin{aligned}
& \max \left\{S z\left(P_{n, 4}^{\prime}\right), S z\left(P_{n}(2,2,3, n-7)\right), S z\left(P_{n}(2,3,2, n-7)\right)\right\} \\
& =\max \left\{\frac{1}{6}\left(n^{3}-19 n+162\right), \frac{1}{6}\left(n^{3}-31 n+306\right), \frac{1}{6}\left(n^{3}-19 n+198\right)\right\} \\
& =\frac{1}{6}\left(n^{3}-19 n+198\right) .
\end{aligned}
$$

Thus $P_{n}(2,3,2, n-7)$ is the unique graph in $\mathbb{U}_{n, 4}$ for $n \geq 10$ with the second largest Szeged index, which is equal to $\frac{1}{6}\left(n^{3}-19 n+198\right)$.

Suppose that $T_{2}$ and $T_{4}$ have more than two vertices. If $t_{2}=3$ and $G \neq P_{n}(2,3,2, n-7)$, then

$$
\begin{aligned}
S z(G) & \leq \max \left\{S z\left(C_{4}\left(P_{2}, S_{3}, P_{2}, P_{n-7}\right)\right), S z\left(C_{4}\left(P_{2}, P_{3}, P_{2}, P_{n-7}^{\prime}\right)\right)\right\} \\
& =\max \left\{\frac{1}{6}\left(n^{3}-25 n+216\right), \frac{1}{6}\left(n^{3}-25 n+216\right)\right\} \\
& =\frac{1}{6}\left(n^{3}-25 n+216\right),
\end{aligned}
$$

where $v_{2}$ is the center of $S_{3}$ and $v_{4}$ is a terminal vertex of $P_{n-7}$ in $C_{4}\left(P_{2}, S_{3}, P_{2}, P_{n-7}\right)$, and $v_{2}$ is a terminal vertex of $P_{3}$ and $v_{4}$ is a terminal vertex of $P_{n-7}^{\prime}$ for $n=11$ and a terminal vertex of $P_{n-7}^{\prime}$ whose neighbor has degree two for $n \geq 12$ in $C_{4}\left(P_{2}, P_{3}, P_{2}, P_{n-7}^{\prime}\right)$. And if $t_{2} \geq 4$, then we have

$$
S z(G) \leq S z\left(P_{n}\left(2, t_{2}, 2, t_{4}\right)\right) \leq S z\left(P_{n}(2,4,2, n-8)\right)=\frac{1}{6}\left(n^{3}-25 n+264\right) .
$$

Thus the graphs in $\mathbb{U}_{n, 4}$ with the third largest Szeged index are just the graphs in $\mathbb{U}_{n, 4} \backslash\left\{P_{n, 4}, P_{n}(2,3,2, n-7)\right\}$ with the largest Szeged index, which is equal to

$$
\begin{gathered}
\max \left\{S z\left(P_{n, 4}^{\prime}\right), S z\left(P_{n}(2,2,3, n-7)\right), S z\left(C_{4}\left(P_{2}, S_{3}, P_{2}, P_{n-7}\right)\right),\right. \\
\left.S z\left(C_{4}\left(P_{2}, P_{3}, P_{2}, P_{n-7}^{\prime}\right)\right), S z\left(P_{n}(2,4,2, n-8)\right)\right\}
\end{gathered}
$$


$=\max \left\{\frac{1}{6}\left(n^{3}-19 n+162\right), \frac{1}{6}\left(n^{3}-31 n+306\right), \frac{1}{6}\left(n^{3}-25 n+216\right), \frac{1}{6}\left(n^{3}-25 n+264\right)\right\}$ i.e., $\frac{1}{6}\left(n^{3}-31 n+306\right)$ for $n=10,11, \frac{1}{6}\left(n^{3}-25 n+264\right)$ for $12 \leq n \leq 16, \frac{1}{6}\left(n^{3}-\right.$ $25 n+264)=\frac{1}{6}\left(n^{3}-19 n+162\right)=792$ for $n=17$, and $\frac{1}{6}\left(n^{3}-19 n+162\right)$ for $n \geq 18$. Thus $P_{n}(2,2,3, n-7)$ for $n=10,11, P_{n}(2,4,2, n-8)$ for $12 \leq n \leq 16$, $P_{17}(2,4,2,9)=P_{17,4}^{\prime}$ for $n=17$, and $P_{n, 4}^{\prime}$ for $n \geq 18$ are the unique graphs with the third largest Szeged index, which is equal to $\frac{1}{6}\left(n^{3}-31 n+306\right)$ for $n=10,11$, $\frac{1}{6}\left(n^{3}-25 n+264\right)$ for $12 \leq n \leq 16,792$ for $n=17$ and $\frac{1}{6}\left(n^{3}-19 n+162\right)$ for $n \geq 18$. The result follows.

Theorem 2. Among the graphs in $\mathbb{U}_{n}$,

(i) $P_{n, 3}$ for $n=6,7$ and $P_{n, 4}$ for $n \geq 8$ are the unique graphs with the largest Szeged index, which is equal to $\frac{1}{6}\left(n^{3}-25 n+96\right)$ for $n=6,7$, and $\frac{1}{6}\left(n^{3}-13 n+144\right)$ for $n \geq 8$

(ii) $S_{7,3}$ for $n=7, P_{8,3}$ for $n=8, S_{9,4}$ for $n=9$, and $P_{n}(2,3,2, n-7)$ for $n \geq 10$ are the unique graphs with the second largest Szeged index, which is equal to 40 for $n=7,68$ for $n=8,120$ for $n=9$, and $\frac{1}{6}\left(n^{3}-19 n+198\right)$ for $n \geq 10$;

(iii) $C_{3}\left(P_{2}, P_{3}, P_{3}\right)$ with $v_{2}$ a terminal vertex of $P_{3}$ and $v_{3}$ a terminal vertex of $P_{3}$ for $n=8, P_{9,3}$ for $n=9, P_{n}(2,2,3, n-7)$ for $n=10,11, P_{n}(2,4,2, n-8)$ for $12 \leq n \leq 16, P_{17}(2,4,2,9)=P_{17,4}^{\prime}$ for $n=17$, and $P_{n, 4}^{\prime}$ for $n \geq 18$ are the unique graphs with the third largest Szeged index, which is equal to 66 for $n=8,100$ for $n=9, \frac{1}{6}\left(n^{3}-31 n+306\right)$ for $n=10,11, \frac{1}{6}\left(n^{3}-25 n+264\right)$ for $12 \leq n \leq 16,792$ for $n=17$, and $\frac{1}{6}\left(n^{3}-19 n+162\right)$ for $n \geq 18$.

Proof. The cases $n=6,7$ are obvious. Suppose that $n \geq 8$. Let $f_{1}(r)=S z\left(P_{n, r}\right)$ if $r$ is odd, and $f_{2}(r)=S z\left(P_{n, r}\right)$ if $r$ is even. If $r$ is odd with $3 \leq r \leq\left\lfloor\frac{n}{2}\right\rfloor-1$, then by Proposition 2,

$$
\begin{aligned}
f_{2}(r+1)-f_{1}(r) & =2 n+3 r^{2}-6 r-1 \geq 2 \cdot 2(r+1)+3 r^{2}-6 r-1 \\
& =3 r^{2}-2 r+3>0,
\end{aligned}
$$

implying that $f_{2}(r+1)>f_{1}(r)$. If $r$ is even with $4 \leq r \leq\left\lfloor\frac{n}{2}\right\rfloor-1$, then by Proposition 2,

$$
\begin{aligned}
f_{1}(r+1)-f_{2}(r) & =(-4 r+2) n+7 r^{2}-4 r \\
& \leq(-4 r+2) \cdot 2(r+1)+7 r^{2}-4 r \\
& =-r^{2}-8 r+4<0,
\end{aligned}
$$

implying that $f_{1}(r+1)<f_{2}(r)$.

For fixed $n$, taking the derivatives for $f_{i}(r)$ where $i=1,2$, whose expressions are given in Proposition 2, we have

$$
f_{1}^{\prime}(r)=\frac{1}{3}\left[15 r^{2}-(6 n+24) r+6 n+7\right],
$$




$$
f_{2}^{\prime}(r)=\frac{1}{3}\left[15 r^{2}-(6 n+36) r+12 n+10\right] .
$$

The two roots of $f_{1}^{\prime}(r)=0$ are $r_{1}$ and $r_{2}$, where

$$
\begin{gathered}
r_{1}=\frac{3 n+12-\sqrt{9(n-1)^{2}+30}}{15}<\frac{3 n+12-3(n-1)}{15}=1<3, \\
r_{2}=\frac{3 n+12+\sqrt{9(n-1)^{2}+30}}{15}>\frac{3 n+12+3(n-1))}{15}=\frac{2}{5} n+\frac{3}{5}>3,
\end{gathered}
$$

and $r_{2}<\left\lfloor\frac{n}{2}\right\rfloor$. Hence, for fixed $n \geq 8, f_{1}^{\prime}(r)<0$ for $3 \leq r<r_{2}$ and $f_{1}^{\prime}(r) \geq 0$ for $r_{2} \leq r \leq\left\lfloor\frac{n}{2}\right\rfloor$. Then, for fixed $n \geq 8, f_{1}(r)$ is decreasing for $3 \leq r<r_{2}$ and increasing for $r_{2} \leq r \leq\left\lfloor\frac{n}{2}\right\rfloor$. The two roots of $f_{2}^{\prime}(r)=0$ are $r_{3}$ and $r_{4}$, where

$$
\begin{gathered}
r_{3}=\frac{3 n+18-\sqrt{9(n-4)^{2}+30}}{15}<\frac{3 n+18-3(n-4)}{15}=2<4, \\
r_{4}=\frac{3 n+18+\sqrt{9(n-4)^{2}+30}}{15}>\frac{3 n+18+3(n-4)}{15}=\frac{2}{5} n+\frac{2}{5} \geq 4
\end{gathered}
$$

for $n \geq 9$, and $r_{4}<\left\lfloor\frac{n}{2}\right\rfloor$ for $n \geq 8$. Hence, for fixed $n \geq 9, f_{2}^{\prime}(r)<0$ for $4 \leq r<r_{4}$ and $f_{2}^{\prime}(r) \geq 0$ for $r_{4} \leq r \leq\left\lfloor\frac{n}{2}\right\rfloor$. Then, for fixed $n \geq 9, f_{2}(r)$ is decreasing for $4 \leq r<r_{4}$ and increasing for $r_{4} \leq r \leq\left\lfloor\frac{n}{2}\right\rfloor$. Note that $\frac{2}{5} n<r_{4}<r_{2}<\frac{2}{5} n+1$ for $n \geq 8$. Let $G \in \mathbb{U}_{n}$.

By Proposition 2 and the properties of $f_{1}(r)$ and $f_{2}(r)$, we have

$$
\begin{aligned}
S z(G) \leq S z\left(P_{n, r}\right) & \leq \max \left\{f_{2}(4), f_{2}\left(\frac{n}{2}\right)\right\} \\
& =\max \left\{\frac{1}{6}\left(n^{3}-13 n+144\right), \frac{1}{8}\left(n^{3}+4 n^{2}-4 n\right)\right\} \\
& =\frac{1}{6}\left(n^{3}-13 n+144\right)
\end{aligned}
$$

if $n \geq 8$ and $n \equiv 0(\bmod 4)$,

$$
\begin{aligned}
S z(G) \leq S z\left(P_{n, r}\right) & \leq \max \left\{f_{2}(4), f_{2}\left(\frac{n-1}{2}\right)\right\} \\
& =\max \left\{\frac{1}{6}\left(n^{3}-13 n+144\right), \frac{1}{8}\left(n^{3}+3 n^{2}+7 n-27\right)\right\} \\
& =\frac{1}{6}\left(n^{3}-13 n+144\right)
\end{aligned}
$$

if $n \geq 9$ and $n \equiv 1(\bmod 4)$,

$$
\begin{aligned}
S z(G) \leq S z\left(P_{n, r}\right) & \leq \max \left\{f_{2}(4), f_{2}\left(\frac{n-2}{2}\right)\right\} \\
& =\max \left\{\frac{1}{6}\left(n^{3}-13 n+144\right), \frac{1}{8}\left(n^{3}+2 n^{2}+24 n-88\right)\right\}
\end{aligned}
$$




$$
=\frac{1}{6}\left(n^{3}-13 n+144\right)
$$

if $n \geq 10$ and $n \equiv 2(\bmod 4)$, and

$$
\begin{aligned}
S z(G) \leq S z\left(P_{n, r}\right) & \leq \max \left\{f_{2}(4), f_{2}\left(\frac{n-3}{2}\right)\right\} \\
& =\max \left\{\frac{1}{6}\left(n^{3}-13 n+144\right), \frac{1}{8}\left(n^{3}+n^{2}+47 n-193\right)\right\} \\
& =\frac{1}{6}\left(n^{3}-13 n+144\right)
\end{aligned}
$$

if $n \geq 11$ and $n \equiv 3(\bmod 4)$. Thus $P_{n, 4}$ is the unique graph in $\mathbb{U}_{n}$ with the largest Szeged index, and then the graphs in $\mathbb{U}_{n}$ with the second largest Szeged index are just the graphs in $\mathbb{U}_{n} \backslash\left\{P_{n, 4}\right\}$ with the largest Szeged index.

Case 1. $n \geq 8$ and $n \equiv 0(\bmod 4)$. The largest Szeged index of graphs in $\mathbb{U}_{n} \backslash\left\{P_{n, 4}\right\}$ is equal to $f_{1}(3)=68$ for $n=8$ and

$$
\begin{gathered}
\max \left\{\operatorname{Sz}\left(P_{n}(2,3,2, n-7)\right), f_{1}(3), f_{2}(6), f_{2}\left(\frac{n}{2}\right)\right\} \\
=\max \left\{\frac{1}{6}\left(n^{3}-19 n+198\right), \frac{1}{6}\left(n^{3}-25 n+96\right), \frac{1}{6}\left(n^{3}-85 n+984\right), \frac{1}{8}\left(n^{3}+4 n^{2}-4 n\right)\right\} \\
=\frac{1}{6}\left(n^{3}-19 n+198\right)
\end{gathered}
$$

for $n \geq 12$. Thus $P_{8,3}$ for $n=8$ and $P_{n}(2,3,2, n-7)$ for $n \geq 12$ are the unique graphs in $\mathbb{U}_{n}$ with the second largest Szeged index. It follows that the graphs in $\mathbb{U}_{n}$ with the third largest Szeged index are just the graphs in $\mathbb{U}_{8} \backslash\left\{P_{8,4}, P_{8,3}\right\}$ for $n=8$ and $\mathbb{U}_{n} \backslash\left\{P_{n, 4}, P_{n}(2,3,2, n-7)\right\}$ for $n \geq 12$ with the largest Szeged index, which is equal to $S z\left(C_{3}\left(P_{2}, P_{3}, P_{3}\right)\right)=66$ for $n=8$, where one $P_{3}$ has a terminal vertex $v_{2}$ and the other $P_{3}$ has a terminal vertex $v_{3}$ in $C_{3}\left(P_{2}, P_{3}, P_{3}\right)$,

$$
\begin{gathered}
\max \left\{S z\left(P_{n}(2,4,2, n-8)\right), f_{1}(3), f_{2}(6), f_{2}\left(\frac{n}{2}\right)\right\} \\
=\max \left\{\frac{1}{6}\left(n^{3}-25 n+264\right), \frac{1}{6}\left(n^{3}-25 n+96\right), \frac{1}{6}\left(n^{3}-85 n+984\right), \frac{1}{8}\left(n^{3}+4 n^{2}-4 n\right)\right\} \\
=\frac{1}{6}\left(n^{3}-25 n+264\right)
\end{gathered}
$$

for $n=12,16$, and

$$
\begin{gathered}
\max \left\{S z\left(P_{n, 4}^{\prime}\right), f_{1}(3), f_{2}(6), f_{2}\left(\frac{n}{2}\right)\right\} \\
=\max \left\{\frac{1}{6}\left(n^{3}-19 n+162\right), \frac{1}{6}\left(n^{3}-25 n+96\right), \frac{1}{6}\left(n^{3}-85 n+984\right), \frac{1}{8}\left(n^{3}+3 n^{2}+7 n-27\right)\right\} \\
=\frac{1}{6}\left(n^{3}-19 n+162\right)
\end{gathered}
$$


for $n \geq 20$. Thus $C_{3}\left(P_{2}, P_{3}, P_{3}\right)$ with $v_{2}$ being a terminal vertex of $P_{3}$ and $v_{3}$ being a terminal vertex of $P_{3}$ for $n=8, P_{n}(2,4,2, n-8)$ for $n=12,16$, and $P_{n, 4}^{\prime}$ for $n \geq 20$ are the unique graphs in $\mathbb{U}_{n}$ with the third largest Szeged index.

Case 2. $n \geq 9$ and $n \equiv 1(\bmod 4)$. The largest Szeged index of graphs in $\mathbb{U}_{n} \backslash\left\{P_{n, 4}\right\}$ is equal to $\max \left\{S z\left(S_{9,4}\right), f_{1}(3)\right\}=\max \{120,100\}=120$ for $n=9$ and

$$
\begin{gathered}
\max \left\{\operatorname{Sz}\left(P_{n}(2,3,2, n-7)\right), f_{1}(3), f_{2}(6), f_{2}\left(\frac{n-1}{2}\right)\right\} \\
=\max \left\{\frac{1}{6}\left(n^{3}-19 n+198\right), \frac{1}{6}\left(n^{3}-25 n+96\right), \frac{1}{6}\left(n^{3}-85 n+984\right), \frac{1}{8}\left(n^{3}+3 n^{2}+7 n-27\right)\right\} \\
=\frac{1}{6}\left(n^{3}-19 n+198\right)
\end{gathered}
$$

for $n \geq 13$. Thus $S_{9,4}$ for $n=9$ and $P_{n}(2,3,2, n-7)$ for $n \geq 13$ are the unique graphs in $\mathbb{U}_{n}$ with the second largest Szeged index. Then the graphs in $\mathbb{U}_{n}$ with the third largest Szeged index are just the graphs in $\mathbb{U}_{9} \backslash\left\{P_{9,4}, S_{9,4}\right\}$ for $n=9$ and $\mathbb{U}_{n} \backslash\left\{P_{n, 4}, P_{n}(2,3,2, n-7)\right\}$ for $n \geq 13$ with the largest Szeged index, which is equal to $S z\left(P_{9,3}\right)=100$ for $n=9$,

$$
\max \left\{S z\left(P_{13}(2,4,2,5)\right), f_{1}(3), f_{2}(6)\right\}=\max \{356,328,346\}=356
$$

for $n=13$,

$$
\begin{gathered}
\max \left\{S z\left(P_{17}(2,4,2,9)\right), S z\left(P_{17,4}^{\prime}\right), f_{1}(3), f_{2}(6), f_{2}(8)\right\} \\
=\max \{792,792,764,742,734\}=792
\end{gathered}
$$

for $n=17$, and

$$
\begin{gathered}
\max \left\{S z\left(P_{n, 4}^{\prime}\right), f_{1}(3), f_{2}(6), f_{2}\left(\frac{n-1}{2}\right)\right\} \\
=\max \left\{\frac{1}{6}\left(n^{3}-19 n+162\right), \frac{1}{6}\left(n^{3}-25 n+96\right), \frac{1}{6}\left(n^{3}-85 n+984\right), \frac{1}{8}\left(n^{3}+3 n^{2}+7 n-27\right)\right\} \\
=\frac{1}{6}\left(n^{3}-19 n+162\right)
\end{gathered}
$$

for $n \geq 21$. Thus $P_{9,3}$ for $n=9, P_{13}(2,4,2,5)$ for $n=13, P_{17}(2,4,2,9)$ and $P_{17,4}^{\prime}$ for $n=17$, and $P_{n, 4}^{\prime}$ for $n \geq 21$ are the unique graphs in $\mathbb{U}_{n}$ with the third largest Szeged index.

Case 3. $n \geq 10$ and $n \equiv 2(\bmod 4)$. The largest Szeged index of graphs in $\mathbb{U}_{n} \backslash$ $\left\{P_{n, 4}\right\}$ is equal to $\max \left\{S z\left(P_{10}(2,3,2,3)\right), f_{1}(3), f_{1}(5)\right\}=\max \{168,141,125\}=$ 168 for $n=10$, and

$$
\begin{gathered}
\max \left\{S z\left(P_{n}(2,3,2, n-7)\right), f_{1}(3), f_{2}(6), f_{2}\left(\frac{n-2}{2}\right)\right\} \\
=\max \left\{\frac{1}{6}\left(n^{3}-19 n+198\right), \frac{1}{6}\left(n^{3}-25 n+96\right), \frac{1}{6}\left(n^{3}-85 n+984\right), \frac{1}{8}\left(n^{3}+2 n^{2}+24 n-88\right)\right\} \\
=\frac{1}{6}\left(n^{3}-19 n+198\right)
\end{gathered}
$$


for $n \geq 14$. Thus for $n \geq 10, P_{n}(2,3,2, n-7)$ is the unique graph in $\mathbb{U}_{n}$ with the second largest Szeged index. Then the graphs in $\mathbb{U}_{n}$ with the third largest Szeged index are just the graphs in $\mathbb{U}_{n} \backslash\left\{P_{n, 4}, P_{n}(2,3,2, n-7)\right\}$ with the largest Szeged index, which is equal to $\max \left\{S z\left(P_{10}(2,2,3,3)\right), f_{1}(3), f_{1}(5)\right\}=\max \{166,141,125\}=166$ for $n=10, \max \left\{S z\left(P_{14}(2,4,2,6)\right), f_{1}(3), f_{2}(6)\right\}=\max \{443,415,423\}=443$ for $n=14$, and

$$
\begin{gathered}
\max \left\{S z\left(P_{n, 4}^{\prime}\right), f_{1}(3), f_{2}(6), f_{2}\left(\frac{n-2}{2}\right)\right\} \\
=\max \left\{\frac{1}{6}\left(n^{3}-19 n+162\right), \frac{1}{6}\left(n^{3}-25 n+96\right), \frac{1}{6}\left(n^{3}-85 n+984\right), \frac{1}{8}\left(n^{3}+2 n^{2}+24 n-88\right)\right\} \\
=\frac{1}{6}\left(n^{3}-19 n+162\right)
\end{gathered}
$$

for $n \geq 18$. Thus $P_{10}(2,2,3,3)$ for $n=10, P_{14}(2,4,2,6)$ for $n=14$, and $P_{n, 4}^{\prime}$ for $n \geq 18$ are the unique graphs in $\mathbb{U}_{n}$ with the third largest Szeged index.

Case 4. $n \geq 11$ and $n \equiv 3(\bmod 4)$. The largest Szeged index of graphs in $\mathbb{U}_{n} \backslash$ $\left\{P_{n, 4}\right\}$ is equal to

$$
\max \left\{S z\left(P_{11}(2,3,2,4)\right), f_{1}(3), f_{1}(5)\right\}=\max \{220,214,164\}=220
$$

for $n=11$, and

$$
\begin{gathered}
\max \left\{S z\left(P_{n}(2,3,2, n-7)\right), f_{1}(3), f_{2}(6), f_{2}\left(\frac{n-3}{2}\right)\right\} \\
=\max \left\{\frac{1}{6}\left(n^{3}-19 n+198\right), \frac{1}{6}\left(n^{3}-25 n+96\right), \frac{1}{6}\left(n^{3}-85 n+984\right), \frac{1}{8}\left(n^{3}+n^{2}+47 n-193\right)\right\} \\
=\frac{1}{6}\left(n^{3}-19 n+198\right)
\end{gathered}
$$

for $n \geq 15$. Thus $P_{n}(2,3,2, n-7)$ for $n \geq 11$ is the unique graph in $\mathbb{U}_{n}$ with the second largest Szeged index. Then the graphs in $\mathbb{U}_{n}$ for $n \geq 11$ with the third largest Szeged index are just the graphs in $\mathbb{U}_{n} \backslash\left\{P_{n, 4}, P_{n}(2,3,2, n-7)\right\}$ with the largest Szeged index, which is equal to

$$
\max \left\{S z\left(P_{11}(2,2,3,4)\right), f_{1}(3), f_{1}(5)\right\}=\max \{216,214,164\}=216
$$

for $n=11, \max \left\{S z\left(P_{15}(2,4,2,7)\right), f_{1}(3), f_{2}(6)\right\}=\max \{544,516,514\}=544$ for $n=15$, and

$$
\begin{gathered}
\max \left\{S z\left(P_{n, 4}^{\prime}\right), f_{1}(3), f_{2}(6), f_{2}\left(\frac{n-3}{2}\right)\right\} \\
=\max \left\{\frac{1}{6}\left(n^{3}-19 n+162\right), \frac{1}{6}\left(n^{3}-25 n+96\right), \frac{1}{6}\left(n^{3}-85 n+984\right), \frac{1}{8}\left(n^{3}+n^{2}+47 n-193\right)\right\} \\
=\frac{1}{6}\left(n^{3}-19 n+162\right)
\end{gathered}
$$

for $n \geq 19$. Thus $P_{11}(2,2,3,4)$ for $n=11, P_{15}(2,4,2,7)$ for $n=15$, and $P_{n, 4}^{\prime}$ for $n \geq 19$ are the unique graphs in $\mathbb{U}_{n}$ with the third largest Szeged index.

By combining Cases $1-4$, the result follows easily. 


\section{ACKNOWLEDGEMENT}

This work was supported by the National Natural Science Foundation of China (No. 11801135, No. 11871239 and No. 11771172) and the China Scholarship Council (No. 201906770057).

\section{REFERENCES}

[1] T. Al-Fozan, P. Manuel, I. Rajasingh, and R. S. Rajan, "A new technique to compute PadmakarIvan index and Szeged index of pericondensed benzenoid graphs," J. Comput. Theor. Nanos., vol. 11, pp. 533-539, 2014, doi: 10.1166/jctn.2014.3390.

[2] K. C. Das and M. J. Nadjafi-Arani, "Comparison between the Szeged index and the eccentric connectivity index," Discrete Appl. Math., vol. 186, pp. 74-86, 2015, doi: 10.1016/j.dam.2015.01.011.

[3] A. A. Dobrynin, "Graphs having maximal value of the Szeged index," Croat. Chem. Acta, vol. 70, pp. 819-825, 1997.

[4] A. A. Dobrynin, "The Szeged and Wiener indices of line graphs," MATCH Commun. Math. Comput. Chem., vol. 79, pp. 743-756, 2018, doi: 10.1016/0893-9659(96)00071-7.

[5] A. A. Dobrynin, R. Entringer, and I. Gutman, "Wiener index of trees: Theory and applications," Acta Appl. Math., vol. 66, pp. 211-249, 2001, doi: 10.1023/A:1010767517079.

[6] A. A. Dobrynin and I. Gutman, "Szeged index of some polycyclic bipartite graphs with circuits of different size," MATCH Commun. Math. Comput. Chem., vol. 35, pp. 117-128, 1997.

[7] I. Gutman, "A formula for the Wiener number of trees and its extension to graphs containing cycles," Graph Theory Notes N. Y., vol. 27, pp. 9-15, 1994.

[8] I. Gutman and O. E. Polansky, Mathematical Concepts in Organic Chemistry. Berlin: SpringerVerlag, 1986.

[9] I. Gutman, L. Popović, P. V. Khadikar, S. Karmarkar, S. Joshi, and M. Mandloi, "Relations between Wiener and Szeged indices of monocyclic molecules," MATCH Commun. Math. Comput. Chem., vol. 35, pp. 91-103, 1997.

[10] I. Gutman, D. Vukičević, and J. Žerovnik, "A class of modified Wiener indices," Croat. Chem. Acta, vol. 77, pp. 103-109, 2004.

[11] P. V. Khadikar, S. Karmarkar, V. K. Agrawal, J. Singh, A. Shrivastava, I. Lukovits, and M. V. Diudea, "Szeged index - Applications for drug modeling," Lett. Drug Design Disc., vol. 2, pp. 606-624, 2005, doi: 10.2174/157018005774717334.

[12] S. Klavžar, A. Rajapakse, and I. Gutman, "The Szeged and the Wiener index of graphs," Appl. Math. Lett., vol. 9, pp. 45-49, 1996, doi: 10.1016/0893-9659(96)00071-7.

[13] J. Li, "A relation between the edge Szeged index and the ordinary Szeged index," MATCH Commun. Math. Comput. Chem., vol. 70, pp. 621-625, 2013.

[14] S. Li and H. Zhang, "Proofs of three conjectures on the quotients of the (revised) Szeged index and Wiener index and beyond," Discrete Math., vol. 340, pp. 311-324, 2017, doi: 10.1016/j.disc.2016.09.007.

[15] M. J. Nadjafi-Arani, H. Khodashenas, and A. R. Ashrafi, "On the differences between Szeged and Wiener indices of graphs,” Discrete Math., vol. 311, pp. 2233-2237, 2011, doi: 10.1016/j.disc.2011.06.019.

[16] S. Simić, I. Gutman, and V. Baltić, "Some graphs with extremal Szeged index," Math. Slovaca, vol. 50, pp. 1-15, 2000.

[17] H. Wiener, "Structural determination of paraffin boiling points," J. Am. Chem. Soc., vol. 69, pp. 17-20, 1947, doi: 10.1021/ja01193a005. 
[18] B. Zhou and X. Cai, "On detour index," MATCH Commun. Math. Comput. Chem., vol. 63, pp. 199-210, 2010.

[19] B. Zhou, X. Cai, and Z. Du, "On Szeged indices of unicyclic graphs," MATCH Commun. Math. Comput. Chem., vol. 63, pp. 113-132, 2010.

Author's address

Xuli Qi

School of Mathematics and Statistics, Central China Normal University, Wuhan 430079, P. R. China E-mail address: qixuli-1212@163.com 